\title{
Effect of habitual exercise on renal carcinogenesis by ferric nitrilotriacetate
}

\author{
Toyohiro Kato $\cdot$ Hiroaki Kawaguchi $\cdot$ Noriaki Miyoshi $\cdot$ \\ Kohji Aoyama - Masaharu Komatsu - Masahisa Horiuchi • \\ Hiroki Yoshida $\cdot$ Toru Takeuchi
}

Received: 29 September 2010/Accepted: 27 October 2010/Published online: 25 November 2010

(C) The Japanese Society for Hygiene 2010

\begin{abstract}
Objectives We investigated whether habitual exercise (HE) (treadmill running) suppresses development of renal cell carcinoma (RCC) induced by ferric nitrilotriacetate (Fe-NTA).

Methods Male Fischer 344 rats were divided into six groups: group I, saline treatment $(12$ weeks $=$ initiation period) and non-HE; group II, Fe-NTA treatment (12 weeks) and non-HE; group III, saline treatment and short-term (12 weeks) HE; group IV, Fe-NTA treatment and short-term HE; group V, saline treatment and long-term (40 weeks) HE; and group VI, Fe-NTA treatment and long-term HE. Saline treatment groups did not develop RCC, therefore we investigated the effects of HE among Fe-NTA treatment groups.
\end{abstract}

T. Kato and H. Kawaguchi contributed equally to this work.

T. Kato $\cdot$ K. Aoyama $\cdot$ M. Komatsu - M. Horiuchi ·

T. Takeuchi $(\square)$

Department of Environmental Medicine, Kagoshima University

Graduate School of Medical and Dental Sciences,

Kagoshima University, 8-35-1 Sakuragaoka,

Kagoshima 890-8544, Japan

e-mail: takeuchi@m.kufm.kagoshima-u.ac.jp

H. Kawaguchi · H. Yoshida

Department of Tumor Pathology, Kagoshima University

Graduate School of Medical and Dental Sciences,

Kagoshima University, Kagoshima, Japan

H. Kawaguchi

Department of Veterinary Experimental Animal Science,

Faculty of Agriculture, Kagoshima University,

Kagoshima, Japan

N. Miyoshi

Department of Veterinary Pathology,

Faculty of Agriculture, Kagoshima University,

Kagoshima, Japan
Results Gross nodules (diagnosed as RCC), RCC represented by microcarcinomas (Mcs), karyomegalic cells (KCs), and degenerative tubules (DTs) were seen in rats treated with Fe-NTA. The number of Mcs, KCs, and DTs were increased in the short-term HE group when compared with those in the non-HE group, but were decreased in the long-term HE group when compared with those in the short-term HE group.

Conclusions Short-term (initiation period) HE promoted renal carcinogenesis induced by Fe-NTA; however, longterm HE after the initiation period suppressed the promoted carcinogenesis.

Keywords Exercise - Ferric nitrilotriacetate $\cdot$ Rat . Renal cell carcinoma $\cdot$ Treadmill running

\section{Introduction}

Habitual exercise (HE) is considered to promote and maintain health [1]. HE has been reported to inhibit induction of breast cancer [2], skin cancer [3], hepatoma [4, 5], colon cancer [6], lymphoma [7], and pancreatic cancer [8], but its effects on renal cancer have received less attention. Conversely, it has been reported that treadmill running stimulates mammary tumorigenesis $[9,10]$. The reason for this discrepancy has not been clarified. There has been speculation that caloric expenditure suppresses cancer development $[3,11,12]$, and it has been suggested that induction of oxidative stress and physical stress enhance carcinogenesis $[9,10]$.

Ferric nitrilotriacetate (Fe-NTA) imposes oxidative stress on the kidney, induces renal proximal tubular damage associated with lipid peroxidation and oxidative DNA base modifications, and finally induces renal cell carcinoma 
(RCC) in rodents [13]. Fe-NTA also induces giant regenerative cells with atypical nuclei of possibly proximal tubular origin (karyomegalic cells, KCs) [14-16].

We previously reported that HE (swimming) induced resistance to oxidative stress, and suppressed 8-hydroxydeoxyguanosine (8OHdG), a typical form of oxidative DNA damage, by Fe-NTA in rat kidney [17]. 8OHdG was reported to correlate well with development of cancer [18, 19]. However, it remains to be determined whether HE inhibits development of RCC.

In this study, we investigated whether HE (treadmill running) suppresses development of RCC induced by Fe-NTA.

\section{Materials and methods}

Animals

Male Fischer 344 rats, aged 5 weeks and with body weight (BW) of 70-90 g, were purchased from Japan SLC, Inc. (Shizuoka, Japan). The animals were maintained in a filtered air laminar flow at the Institute of Laboratory Animal Sciences, Frontier Science Research Center, Kagoshima University. Room temperature was maintained at $25 \pm 2^{\circ} \mathrm{C}$ and relative humidity at $55 \pm 10 \%$, with a $12 \mathrm{~h} \mathrm{light/dark} \mathrm{cycle.} \mathrm{They} \mathrm{were} \mathrm{provided} \mathrm{with} \mathrm{commercial}$ rat chow (CE-2, CLEA Inc., Tokyo, Japan) and tap water, and were used after acclimation for 7 days. After the start of the experiment, sedentary rat groups were fed an amount equivalent to the mean food intake of the exercise groups. The use of animals in this research complied with all relevant guidelines set by Kagoshima University.

\section{Preparation of Fe-NTA solution}

$\mathrm{Fe}\left(\mathrm{NO}_{3}\right)_{3}$ and $\mathrm{Na}_{2} \mathrm{NTA}$ were purchased from Wako Pure Chemical (Osaka, Japan). Fe-NTA solution was prepared immediately before use by the method of Toyokuni et al. [13]; $\mathrm{Fe}\left(\mathrm{NO}_{3}\right)_{3}$ and $\mathrm{Na}_{2} \mathrm{NTA}$ were each dissolved in Milli$\mathrm{Q}$ water and were mixed in molar ratio of 1:4. $\mathrm{pH}$ was adjusted to 7.4 with $\mathrm{NaHCO}_{3}$.

\section{Study design}

Rats were divided into six groups (Fig. 1): group I, saline treatment and nonexercise $(n=8)$; group II, Fe-NTA treatment and nonexercise $(n=8)$; group III, saline treatment and short-term exercise $(n=8)$; group IV, Fe-NTA treatment and short-term exercise $(n=8)$; group $\mathrm{V}$, saline treatment and long-term exercise $(n=7)$; and group VI, Fe-NTA treatment and long-term exercise $(n=8)$. The short-term exercise regimen was 12 weeks treadmill running with Rat Runner RR-1200 (Agawa Machine Inc., Shimane, Japan). The

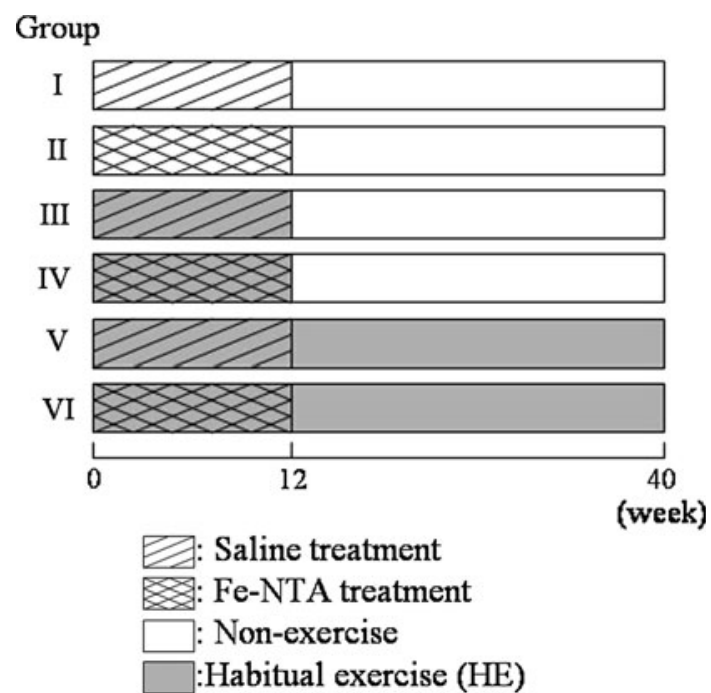

Fig. 1 Summary of the experimental protocol

long-term exercise regimen was 40 weeks treadmill running. Fe-NTA or saline was administered once a day by i.p. injection $(5 \mathrm{mg} \mathrm{Fe} / \mathrm{kg} \mathrm{BW})$ for the initial 7 days, and was then administered 3 times per week $(10 \mathrm{mg} \mathrm{Fe} / \mathrm{kg} \mathrm{BW})$ for 11 weeks. Treadmill training $(8 \mathrm{~m} / \mathrm{min}$ at $0 \%$ incline $)$ was carried out for $15 \mathrm{~min}$ before injection up to 12 weeks (groups III, IV, V, and VI). Group V and VI rats were then trained up to 24 weeks, $8 \mathrm{~m} / \mathrm{min}$ at $0 \%$ incline for $30 \mathrm{~min}$, 5 days/week. Due to a decline in exercise ability, the exercise intensity was decreased afterwards, i.e., up to 39 weeks, $8 \mathrm{~m} / \mathrm{min}$ at $0 \%$ incline for $15 \mathrm{~min}, 5$ days/week; and up to 40 weeks, $8 \mathrm{~m} / \mathrm{min}$ at $0 \%$ incline for $15 \mathrm{~min}, 3$ days/week.

Necropsy and gross examination

At the end of the experiment, all animals were anesthetized and killed by exsanguination through the abdominal aorta. At necropsy, the surface of the bilateral kidneys was grossly examined for nodules, the kidneys were cut to thickness of $2-3 \mathrm{~mm}$, and transections were then grossly examined for nodules. The longest and shortest diameters of all nodules were measured, and their lengths multiplied together to calculate the area $\left(\mathrm{mm}^{2}\right)$. The area of each nodule is expressed as $\mathrm{mm}^{2}$ /nodule. Each animal, and the bilateral kidneys and adrenal glands, were weighed.

We initially planned six groups, but RCC was not found in the saline treatment groups. Therefore, we investigated the effects of HE on development of RCC in the Fe-NTA treatment groups (II, IV, and VI).

\section{Histopathological examination}

The kidneys were fixed in $10 \%$ phosphate-buffered formalin, dehydrated, and then embedded in paraffin. The 


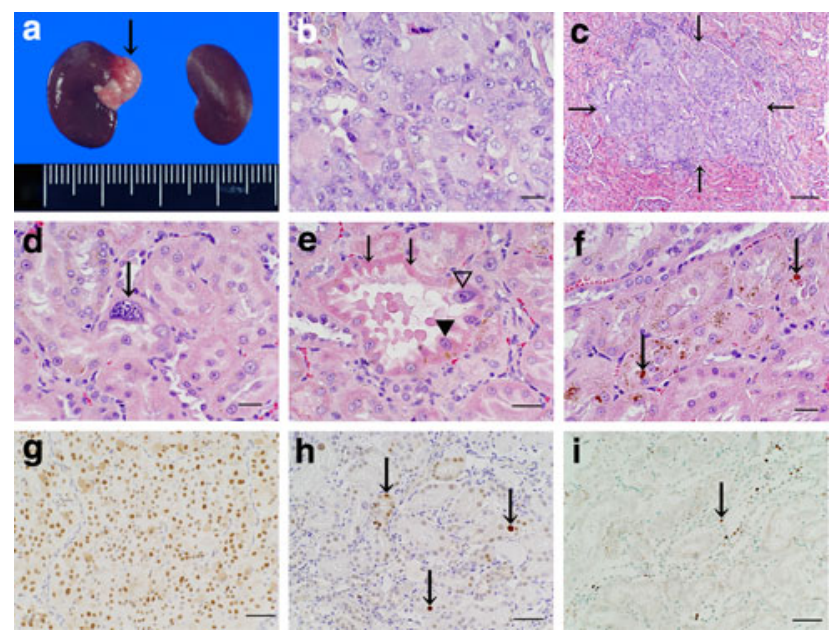

Fig. 2 a White nodule (down arrow) induced in kidney by Fe-NTA in a group II rat. b Renal cell carcinoma induced in kidney by Fe-NTA in a group VI rat. $\mathbf{c}$ Renal cell microcarcinoma (down arrow) induced in kidney by Fe-NTA in a group VI rat. d Karyomegalic cell (down arrow) induced in proximal tubule by Fe-NTA in a group II rat. e Degenerative tubule with eosinophilic cytoplasm (down arrows), atypia (filled inverted triangle), and karyomegalic cell (open inverted triangle) induced in kidney by Fe-NTA in a group II rat. f Brown droplets (down arrows) in proximal tubule in a group II rat. $\mathbf{g}$ PCNA-positive nuclei in a renal cell carcinoma in a group II rat. h PCNA-positive nuclei (down arrows) in some proximal tubule epithelial cells in a group VI rat. i TUNEL-positive nuclei (down arrow) in some proximal tubule epithelial cells in a group VI rat. Bar $100 \mu \mathrm{m}(\mathbf{c}), 50 \mu \mathrm{m}(\mathbf{e}, \mathbf{g}-\mathbf{i}), 20 \mu \mathrm{m}(\mathbf{b}, \mathbf{d}, \mathbf{f}) . \mathrm{H} \& \mathrm{E}$ stain: b-f; Immunohistochemistry for PCNA $(\mathbf{g}, \mathbf{h})$ and apoptotic cells by TUNEL (i)

maximum transection surface of the kidney (center of the kidney) and gross nodules were sectioned to $5 \mu \mathrm{m}$, stained routinely with hematoxylin and eosin (H\&E) stain, and then examined histopathologically. The numbers of microcarcinomas (Mcs), KCs, degenerative tubules (DTs), proximal tubules (PTs) with brown droplet (BD), and BDs in the maximum transection surface were counted. All Mcs in the maximum transection surface were $<1 \mathrm{~mm}$ in diameter and could not be grossly observed, but features of carcinoma, such as atypia and duct, and relatively clear border were microscopically observed in Mcs (Fig. 2c). The areas $\left(\mathrm{mm}^{2}\right)$ of Mcs were also measured by Image Processor for Analytical Pathology (IPAP; Sumika Technoservice Corporation, Hyogo, Japan).

Kidney sections were stained histochemically by Berlin blue (BB), Turnbull blue (TB), periodic acid Schiff (PAS), and Schmorl stain for BDs in PTs.

\section{Immunohistochemistry for PCNA}

Deparaffinized kidney sections were examined immunohistochemically. After endogenous peroxidase activity had been blocked with $2 \%$ hydrogen peroxide, the sections were pretreated with $10 \mathrm{mM}$ citrate buffer $(\mathrm{pH}$ 6.0) by microwaves. After rinsing with phosphate-buffered saline (PBS), the sections were stained with Zymed's proliferating cell nuclear antigen (PCNA) staining kit (Zymed Laboratories Inc., San Francisco, CA) according to the manufacturer's instructions. The sections were then visualized with VECTASTAIN Elite ABC kit (Vector Laboratories, USA) according to the manufacturer's instructions. The sections were then washed, counterstained with hematoxylin, dehydrated, cleared in xylene, and mounted. RCC diagnosed in this study was used as a positive control for PCNA, and cells with stained nuclei in PTs were interpreted as positive. PCNA-stained cell nuclei were counted in PTs of five microscopic fields $\left(1\right.$ field = approximately $\left.0.148 \mathrm{~mm}^{2}\right)$ selected randomly. Mean numbers of positive nuclei in the five microscopic fields were calculated. Each PT contained approximately 8-24 epithelial cells.

Immunohistochemistry for apoptotic cells by TUNEL

Deparaffinized kidney sections from four animals selected randomly from each group were treated with proteinase $\mathrm{K}$ $(20 \mu \mathrm{g} / \mathrm{ml})$ for $15 \mathrm{~min}$ at room temperature, and then endogenous peroxidase was blocked with $2 \%$ hydrogen peroxidase. The sections were stained with Apop Tag Plus peroxidase in situ apoptosis detection kit (Intergen, Purchase, NY) according to the manufacturer's instructions. The positive section in the kit was used as a positive control for terminal deoxynucleotidyl transferase dUTP nick end labeling (TUNEL), and cells with stained nuclei were interpreted as positive in PTs. Immunostained cell nuclei were counted in PTs in five microscopic fields $\left(1\right.$ field $=$ approximately $0.148 \mathrm{~mm}^{2}$ ) selected randomly.

\section{Statistics}

Mean differences were evaluated by least significant difference (LSD). Data are shown as mean \pm standard deviation (SD). Incidences (percentages) of rats with gross nodule, Mc, and $\mathrm{KC}$ were tested using a fourfold contingency table (chi-square test); $p<0.05$ was considered statistically significant.

\section{Results}

Gross examination

As shown in Table 1 , BW of rats in group VI $(p<0.05)$ was significantly lower than that in group II. Total food intake during the experimental period per rat of group II, IV, and VI was 4168.8, 4311.5, and $4367.4 \mathrm{~g}$, respectively. The absolute weight (AW) of the adrenal glands in 
Table 1 Effects of habitual exercise on body weight and weight of adrenal glands and kidney at necropsy

\begin{tabular}{lccc}
\hline & Group II $(n=8)$ & Group IV $(n=8)$ & Group VI $(n=7)$ \\
\hline BW (g) & $372.5 \pm 19.8$ & $366.0 \pm 19.6$ & $350.0 \pm 12.8^{\mathrm{a}}$ \\
Adrenal glands & & & \\
AW (mg) & $40.0 \pm 5.8$ & $37.8 \pm 3.7$ & $45.9 \pm 6.3^{\mathrm{a}, \mathrm{bb}}$ \\
AW/BW (mg/g) & $0.108 \pm 0.015$ & $0.103 \pm 0.012$ & $0.132 \pm 0.021^{\text {aa,bb }}$ \\
Kidneys & & & $2.14 \pm 0.31$ \\
AW (g) & $2.08 \pm 0.17$ & $5.9 \pm 0.8$ & $2.07 \pm 0.14$ \\
AW/BW (mg/g) & $5.6 \pm 0.3$ & $5.9 \pm 0.4$ \\
\hline
\end{tabular}

$B W$ body weight, $A W$ absolute weight

${ }^{\mathrm{a}} p<0.05,{ }^{\text {aa }} p<0.01$ : significantly different from group II

bb $p<0.01$ : significantly different from group IV

Table 2 Gross and histopathological examination in the kidney

\begin{tabular}{llll}
\hline & Group II $(n=8)$ & Group IV $(n=8)$ & Group VI $(n=7)$ \\
\hline Number of gross nodules (diagnosed as RCC) & 3 & 1 & 2 \\
Number of rats with gross nodule $(\%)$ & $2(25.0 \%)$ & $1(12.5 \%)$ & $2(28.6 \%)$ \\
Number of gross nodules/rat & $0.38 \pm 0.74$ & $0.13 \pm 0.35$ & $0.29 \pm 0.00$ \\
Mean size of gross nodule $\left(\mathrm{mm}^{2}\right)$ & $3.7 \pm 2.2$ & 54.0 & $13.3 \pm 16.6$ \\
Number of rats with Mc $(\%)$ & $2(25.0 \%)$ & $7(87.5 \%)^{\mathrm{a}}$ & $2(28.6 \%)^{\mathrm{b}}$ \\
Number of Mc & 2 & 12 & 2 \\
Number of Mc/rat & $0.25 \pm 0.46$ & $1.05 \pm 0.93^{\text {aa }}$ & $0.29 \pm 0.49^{\text {bb }}$ \\
Mean size of Mc (mm $\left.{ }^{2}\right)$ & $0.14 \pm 0.05$ & $0.11 \pm 0.17$ & $0.20 \pm 0.03$ \\
Number of rats with KC $(\%)$ & $8(100 \%)$ & $8(100 \%)$ & $7(100 \%)$ \\
Number of KC/rat & $11.5 \pm 5.4$ & $28.1 \pm 12.9^{\text {aa }}$ & $15.7 \pm 8.2^{\text {bb }}$ \\
Number of rats with DT $(\%)$ & $6(75.0 \%)$ & $8(100 \%)$ & $6(85.7 \%)$ \\
Number of DT/rat & $1.13 \pm 0.99$ & $3.50 \pm 2.62^{\text {aa }}$ & $2.14 \pm 1.21$ \\
Number of PTs with BDs & $61.3 \pm 13.9$ & $52.1 \pm 5.9$ & $34.3 \pm 17.3^{\text {aa,bb }}$ \\
Number of BDs & $86.0 \pm 22.7$ & $82.0 \pm 9.4$ & $43.4 \pm 12.1^{\text {aa,bb }}$ \\
\hline
\end{tabular}

$R C C$ renal cell carcinoma, $M c$ microcarcinoma, $K C$ karyomegalic cell, $D T$ degenerative tubule, $P T$ proximal tubule, $B D$ brown droplet

${ }^{\mathrm{a}} p<0.05,{ }^{\text {aa }} p<0.01$ : significantly different from group II

${ }^{\mathrm{b}} p<0.05,{ }^{\mathrm{bb}} p<0.01$ : significantly different from group IV

group VI was significantly higher than that in groups II $(p<0.05)$ and IV $(p<0.01)$, and AW/BW in group VI was significantly higher than that in groups II and IV (both $p<0.01)$. No differences were found in AW or AW/BW of kidneys among the Fe-NTA treatment groups. Single or multiple white nodules were observed in the kidneys of Fe-NTA treatment groups (Fig. 2a). All nodules were microscopically diagnosed as RCC (Fig. 2b) as described previously [20]. No significant differences in the number of rats with nodules, number of nodules, number of nodules per rat, or mean area of nodules were found among the FeNTA treatment groups (Table 2). One rat in group VI died at week 39 after the experiment due to bleeding from a large RCC.
Histopathological examination

As shown in Table 2, Mcs were observed in only the Fe-NTA treatment groups (Fig. 2c). The number of rats with Mcs in group IV was significantly higher than that in groups II and VI (both $p<0.05$ ). The number of Mcs per rat in group IV was significantly higher than that in groups II and VI (both $p<0.01$ ).

KCs were seen in PTs and DTs (Fig. 2d). KCs were observed in only the Fe-NTA treatment groups. The number of KCs per rat in group IV was significantly higher than that in groups II and VI (both $p<0.01$ ).

DTs with atypia and eosinophilic cytoplasm were observed in only the Fe-NTA treatment groups (Fig. 2e). 
Table 3 Immunohistochemistry for PCNA and apoptotic cells by TUNEL

\begin{tabular}{llll}
\hline & Group II & Group IV & Group VI \\
\hline Number of PCNA-positive cells in PTs & $3.46 \pm 1.95(n=8)$ & $4.13 \pm 2.03(n=8)$ & $1.90 \pm 1.01^{\mathrm{b}}(n=7)$ \\
Number of TUNEL-positive cells in PTs & $1.50 \pm 0.19(n=4)$ & $2.58 \pm 0.58^{\text {aa }}(n=4)$ & $1.42 \pm 0.32^{\text {bb }}(n=4)$ \\
\hline
\end{tabular}

$P T$ proximal tubule

${ }^{\text {aa }} p<0.01$ : significantly different from group II

${ }^{\mathrm{b}} p<0.05,{ }^{\mathrm{bb}} p<0.01$ : significantly different from group IV

Some DTs had one, or a few KCs. The number of DTs per rat in group IV was significantly higher than that in group II $(p<0.01)$.

BDs were observed in PTs in both the saline and Fe-NTA treatment groups (Fig. 2f). However, the number of PTs with BDs in the Fe-NTA treatment groups was significantly higher than that in saline treatment groups $(p<0.01)$. The number of PTs with BDs and BDs in group VI was significantly lower than that in groups II and IV (all $p<0.01$ ). BDs were histochemically negative for $\mathrm{BB}$ and $\mathrm{TB}$ stains, and some of the BDs were slightly positive for PAS and Schmorl stains.

Immunohistochemical examination

A large number of PCNA-positive cells were observed in RCCs (Fig. 2g). As shown in Table 3, the number of PCNA-positive cells in PTs (Fig. 2h) in group IV was significantly higher than that in group VI $(p<0.05)$. As shown in Table 3, the number of TUNEL-positive cells in PTs (Fig. 2i) in group IV was significantly higher than that in groups II and VI (both $p<0.01$ ).

\section{Discussion}

HE has been reported both to prevent [2-8] and to promote $[9,10]$ cancer development. HE increases oxygen consumption and may also increase generation of reactive oxygen species (ROS) in the body during exercise [21]. However, we and others have reported that HE increases resistance to oxidative stress $[17,21]$. ROS are considered to induce and promote cancer [22]. Fe-NTA, a renal carcinogen, imposes oxidative stress on the kidney and induces oxidative DNA damage [13-15, 17]. In this study, we investigated the effects of $\mathrm{HE}$, which may modulate generation of ROS, on ROS-related carcinogenesis, i.e., Fe-NTA-induced RCC [13].

We imposed short-term or long-term HE on rats. Shortterm HE was imposed during the Fe-NTA injection period. The period was regarded as the initiation period, because oxidative DNA damage has been reported to increase during such injection [14, 15]. Long-term HE was imposed both during and after injection. We reported previously on a swimming regimen [17], but in this study we employed treadmill running, because swimming could be fatal to rats.

Rats on long-term HE showed lower BW than those in other groups despite similar food intake. This finding suggests that HE provided sufficient physical activity to suppress BW. However, long-term HE might have induced psychological stress, because adrenal gland weight was increased [23].

Neither short-term nor long-term HE affected development of gross nodules. However, short-term HE increased the number of $\mathrm{Mc}$ and $\mathrm{KC}$. $\mathrm{KC}$ is considered a precancerous stage of cancer [14-16]. Renal blood flow is reported to decrease to $20 \%$ during exercise, returning to a resting state after exercise [24]. This is similar to ischemiareperfusion and may increase generation of ROS after exercise [24]. We injected Fe-NTA after exercise. Fe-NTA, together with increased ROS, might have induced more carcinogenic oxidative DNA and lipid damage $[14,15]$ in the HE groups than in the sedentary group, and might also have increased the numbers of $\mathrm{Mc}$ and $\mathrm{KC}$ in the shortterm HE group. Long-term HE, i.e., HE after the Fe-NTA injection period, suppressed the numbers of $\mathrm{Mc}$ and $\mathrm{KC}$ increased by short-term $\mathrm{HE}$ and also suppressed the numbers of apoptotic cells and PCNA-positive cells. Apoptosis may provide spaces for proliferation of initiated cells. Long-term HE might have increased resistance to oxidative stress $[17,21]$ and at the same time might have reduced susceptibility to apoptosis [25, 26].

Long-term HE also decreased the number of DTs, which might be considered as precancerous because DTs contain atypical cells and KCs. However, the reasons why DTs were increased in short-term HE group were not clear, thus further study is required to clarify the mechanisms of DT induction.

We found BDs in both the saline treatment and Fe-NTA treatment groups. However, the saline treatment group showed considerably fewer BDs $(6.1 \pm 3.2$ in group I) than the Fe-NTA groups. BDs were stained weakly with PAS and Schmorl staining, indicating the presence of lipofuscin, a lipid degeneration product. The long-term HE 
group showed significantly fewer BDs than the sedentary and short-term HE groups. Long-term HE possibly reduced lipid degeneration due to increased oxidative stress resistance.

In conclusion, this is the first report to investigate the effects of HE on ROS-related carcinogenesis. Exercise during exposure to an ROS-generating carcinogen, i.e., exercise during the initiation period, increased the number of early and precancerous stages of cancer. In contrast, long-term HE after the initiation period might have suppressed cancer development. HE employed in this report might have imposed psychological stress due to forced treadmill running. Psychological stress is known to reduce natural killer cell activity $[27,28]$, resulting in possible increase in cancer development. Although the long-term $\mathrm{HE}$ in this report showed beneficial effects on cancer suppression, the benefits might have been weakened by psychological stress. HE with less stress or free from stress, such as voluntary HE, may show clearer benefits for cancer prevention than forced HE. Studies on prevention of cancer by long-term voluntary $\mathrm{HE}$ are warranted.

Acknowledgments We are grateful to Dr. S. Toyokuni (Nagoya University Graduate School of Medicine) for his valuable advice, and to Mr. T. Kodama Mr. M. Souda, Ms. C. Yumiba (Kagoshima University Graduate School of Medical and Dental Sciences), and Mr. G. Martin (Shin Nippon Biomedical Laboratories, Ltd., Kagoshima, Japan) for their valuable technical assistance. Parts of the work were supported by Grand-in-Aids for Scientific Research awarded by the Ministry of Education, Science, Sports, and Culture of Japan (\#14370140 and \#16659149).

\section{References}

1. Centers for Disease Control, National Center for Chronic Disease Prevention, Health Promotion. Physical activity and good nutrition: essential elements to prevent chronic diseases and obesity 2003. Nutr Clin Care. 2003;6:135-8.

2. Westerlind KC, McCarty HL, Schultheiss PC, Story R, Reed AH, Baier ML, et al. Moderate exercise training slows mammary tumour growth in adolescent rats. Eur J Cancer Prev. 2003;12:281-7.

3. Michna L, Wagner GC, Lou YR, Xie JG, Peng QY, Lin Y, et al. Inhibitory effects of voluntary running wheel exercise on UVBinduced skin carcinogenesis in SKH-1 mice. Carcinogenesis. 2006;27:2108-15.

4. Ikuyama T, Watanabe T, Minegishi Y, Osanai H. Effect of voluntary exercise on 3-methyl-4-dimethylaminoazobenzeneinduced hepatomas in male Jc1:Wistar rats. Proc Soc Exp Biol Med. 1993;204:211-5.

5. Sugie S, Reddy BS, Lowenfels A, Tanaka T, Mori H. Effect of voluntary exercise on azoxymrthane-induced hepayocarcinogenesis in male F344 rats. Cancer Lett. 1992;63:67-72.

6. Reddy BS, Sugie S, Lowenfels A. Effect of voluntary exercise on azoxymethane-induced colon carcinogenesis in male F344 rats. Cancer Res. 1988;48:7079-81.

7. Singh MP, Singh G, Singh SM. Role of host's antitumor immunity in exercise-dependent regression of murine T-cell lymphoma. Comp Immunol Microbiol Infect Dis. 2005;28:231-48.
8. Roebuck BD, McCaffrey J, Baumgartner K. Protective effects of voluntary exercise during the postinitiation phase of pancreatic carcinogenesis in the rat. Cancer Res. 1990;50:6811-6.

9. Thompson HJ, Ronan AM, Ritacco KA, Tagliaferro AR. Effect of type and amount of dietary fat on the enhancement of rat mammary tumorigenesis by exercise. Cancer Res. 1989;49: 1904-8.

10. Thompson HJ, Ronan AM, Ritacco KA, Tagliaferro AR, Meeker LD. Effect of exercise on the induction of mammary carcinogenesis. Cancer Res. 1988;48:2720-3.

11. Colbert LH, Mai V, Tooze JA, Perkins SN, Berrigan D, Hursting $\mathrm{SD}$. Negative energy balance induced by voluntary wheel running inhibits polyp development in APCMin mice. Carcinogenesis. 2006;27:2103-7.

12. Craven-Giles T, Tagliaferro AR, Ronan AM, Baumgatner AM, Baumgartner KJ, Roebuck BD. Dietary modulation of pancreatic carcinogenesis: calories and energy expenditure. Cancer Res. 1994;54:1964s-8s.

13. Toyokuni S, Mori T, Hiai H, Dizdaroglu M. Treatment of Wistar rats with a renal carcinogen, ferric nitrilotriacetate, causes DNAprotein cross-linking between thymine and tyrosine in their renal chromatin. Int J Cancer. 1995;62:309-13.

14. Shimoi K, Shen B, Toyokuni S, Mochizuki R, Furugori M, Kinae N. Protection by alpha G-rutin, a water-soluble antioxidant flavonoid, against renal damage in mice treated with ferric nitrilotriacetate. Jpn J Cancer Res. 1997;88:453-60.

15. Toyokuni S, Mori T, Dizdaroglu M. DNA base modifications in renal chromatin of Wistar rats treated with a renal carcinogen, ferric nitrilotriacetate. Int J Cancer. 1994;57:123-8.

16. Tanaka T, Akatsuka S, Ozeki M, Shirase T, Hiai H, Toyokuni S. Redox regulation of annexin 2 and its implications for oxidative stress-induced renal carcinogenesis and metastasis. Oncogene. 2004;23:3980-9.

17. Nakatani K, Komatsu M, Kato T, Yamanaka T, Takekura H, Wagatsuma A, et al. Habitual exercise induced resistance to oxidative stress. Free Radic Res. 2005;39:905-11.

18. Halliwell B. Oxygen and nitrogen are pro-carcinogens. Damage to DNA by reactive oxygen, chlorine and nitrogen species: measurement, mechanism and the effects of nutrition. Mutat Res. 1999;443:37-52.

19. Kasai H. Chemistry-based studies on oxidative DNA damage: formation, repair, and mutagenesis. Free Radic Biol Med. 2002; 33:450-6.

20. Ebina Y, Okada S, Hamazaki S, Ogino F, Li JL, Midorikawa O. Nephrotoxicity and renal cell carcinoma after use of iron- and aluminum-nitrilotriacetate complexes in rats. J Natl Cancer Inst. 1986;76:107-13.

21. Powers SK, Ji LL, Leeuwenburgh C. Exercise training-induced alterations in skeletal muscle antioxidant capacity: a brief review. Med Sci Sports Exerc. 1999;31:987-97.

22. Beckman KB, Ames BN. Oxidative decay of DNA. J Biol Chem. 1997;272:19633-6.

23. Welch BL, Welch AS. Sustained effects of brief daily stress (fighting) upon brain and adrenal catecholamines and adrenal spleen, and heart weights of mice. Proc Natl Acad Sci USA. 1969;64:100-7.

24. Maeda S, Miyauchi T, Iemitsu M, Tanabe T, Irukayama-Tomobe $\mathrm{Y}$, Goto $\mathrm{K}$, et al. Involvement of endogenous endothelin-1 in exercise-induced redistribution of tissue blood flow: an endothelin receptor antagonist reduces the redistribution. Circulation. 2002;106:2188-93.

25. Liang H, Hilton G, Mortensen J, Regner K, Johnson CP, Nilakantan V. MnTmPyP, a cell permeable SOD mimetic, reduces oxidative stress and apoptosis following renal ischemia-reperfusion. Am J Physiol Renal Physiol. 2009;296:F226-76. 
26. Powers SK, Lennon SL. Analysis of cellular responses to free radicals: focus on exercise and skeletal muscle. Proc Nutr Soc. 1999;58:1025-33.

27. Wu W, Yamaura T, Murakami K, Murata J, Matsumoto K, Watanabe $\mathrm{H}$, et al. Social isolation stress enhanced liver metastasis of murine colon 26-L5 carcinoma cells by suppressing immune responses in mice. Life Sci. 2000;66:1827-38.
28. Yermal SJ, Witek-Janusek L, Peterson J, Mathews HL. Perioperative pain, psychological distress, and immune function in men undergoing prostatectomy for cancer of the prostate. Biol Res Nurs. 2010;11:351-62. 